\title{
Management aus einem Guss
}

VON PETRA GERBRACHT, FRIEDHART HEGNER UND ULRICH KRAMER

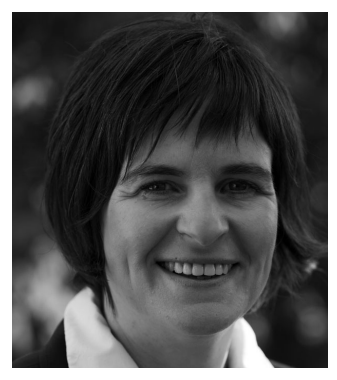

Petra Gerbracht ist Projektleiterin im Institut für Sozialplanung, Management und Verwaltung (ISMV) Dr. Hegner \& Partner GmbH mit Sitz in Berlin und Bielefeld.

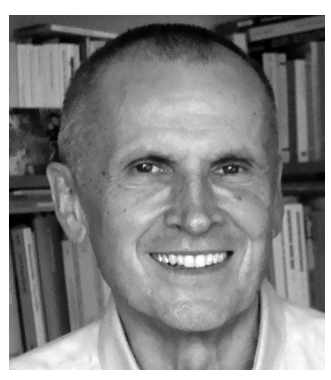

Dr. Friedhart Hegner ist zusammen mit Ulrich Kramer geschäftsführende Gesellschafter der ISMV Dr. Hegner \& Partner $\mathrm{GmbH}$.

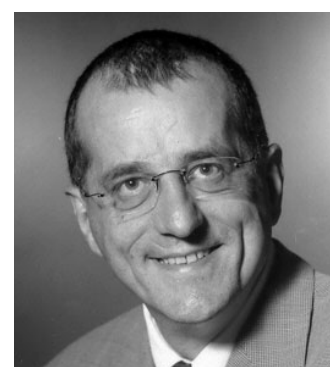

Ulrich Kramer ist zusammen mit Dr. Friedhart Hegner geschäftsführende Gesellschafter der ISMV Dr. Hegner \& Partner $\mathrm{GmbH}$. www.ismv.de

\author{
Organisations- und Personalentwicklung müssen \\ aufeinander abgestimmt sein, wenn sie zum \\ Unternehmenserfolg beitragen sollen. Das kann \\ jedoch auf Dauer nur gelingen, wenn beide auf \\ einer gemeinsamen Wertorientierung fußen.
}

Personal- und Organisationsentwicklung wirkt nur dann nachhaltig als Erfolgsfaktor, wenn die Maßnahmen gut koordiniert sind, und zwar sowohl die Zielsetzungen als auch die Umsetzungsschritte. Angesichts der Vielfalt sozial- und gesundheitswirtschaftlicher Unternehmen sind für die Praxis zwei Konstellationen handlungsrelevant:

- größere Unternehmen, Träger und Einrichtungen mit eigenen Stabsstellen für Organisationsentwicklung und Personalentwicklung, teils als Spezialstellen ausdifferenziert und teils in einer Stabsstelle beim Vorstand oder bei der Geschäftsführung angesiedelt

- kleinere und mittlere Unternehmen, bei denen Aufgaben der Personalund Organisationsentwicklung von einzelnen Mitarbeitenden neben anderen Arbeiten zu erledigen sind, beispielsweise von der Personalleitung, von der Geschäftsführung oder von einzelnen Bereichsleitungen.

Im ersten Fall geht es beim Integrieren beider Aufgabenfelder darum

- die Arbeit der Stabsstellen zu koordinieren, was zu den Leitungsaufgaben gehört, sowie

- deren Arbeit an andere Aktivitäten der Unternehmensentwicklung »anzudocken $«$.

Im zweiten Fall kommt es darauf an, im Kurz- und Langzeitgedächtnis sowie in den Handlungsroutinen der jeweils Verantwortlichen zu verankern, dass
Organisationsentwicklung und Personalentwicklung bei der Beschäftigung mit anderen Maßnahmen nicht ausgeblendet werden.

\section{Drei Kernfragen der Unternehmensentwicklung}

Bei den anderen Maßnahmen der Unternehmensentwicklung sind insbesondere solche Schritte erforderlich, durch die Personal- und Organisationsentwicklung mit der Ausarbeitung und Umsetzung strategischer Konzepte und eines Unternehmensleitbilds verbunden werden. Dabei wird die Planung und Entwicklung der Angebotspalette häufig zu spät hinsichtlich der Konsequenzen für Organisations- und Personalstruktur reflektiert. Demgegenüber verhindert die frühzeitige Beantwortung dreier Fragen spätere Friktionen:

1.

Was sind heute und zukünftig die - relevanten Adressatengruppen des Unternehmens, also die Mittelgeber (z. B. öffentliche Hand, Sponsoren) und die Kunden, Betreuten, Patienten, Klienten usw. mit den jeweils zu befriedigenden Bedürfnissen?

7 Wo liegen die heutigen und zu- künftigen Schwerpunkte der Angebotspalette, also der Dienst- und Sachleistungen, die qualitätssicher und termintreu zu erbringen und an die Adressaten $\mathrm{zu}$ übermitteln sind?

3 Welchen "Innenausbau« (fach3. sprachlich: Organisationstechnologie) benötigen die Einrichtung und 
der Träger, um treffsicher mit geeigneten Angeboten auf die Anliegen der Adressaten reagieren zu können?

Die drei Komponenten bilden das Aktionsfeld, auf dem sich alle Aktivitäten der Unternehmensangehörigen bewegen. Der Erfolg des Unternehmens hängt davon ab, wie gut die drei Komponenten durch koordinierte Maßnahmen der Organisations- und Personalentwicklung aufeinander abgestimmt sind. Misslingt die Abstimmung der Komponenten, kommt es zu einer - längerfristig gefährlichen - Schieflage des Unternehmens im Wettbewerbsfeld. Gemeint ist damit die Relation zwischen - nach außen geblickt - Preisniveau und Angebotsqualität sowie - mit Blick nach innen - die Relation zwischen Kostenniveau und Qualitätssicherung. stabilisiertes Vorgehen ermöglichen, wenn es darum geht, Personal anzuwerben, auszuwählen, einzuarbeiten, zu qualifizieren (inklusive Fort- und Weiterbildung) sowie zu motivieren, aber auch sich notfalls von Mitarbeitenden zu trennen. Daraus resultiert die Sozialstruktur der Belegschaft, also Altersaufbau, Verteilung von Männern und Frauen auf die Hierarchieebenen, Immigrantenanteil etc.

Blickt man vor diesem Hintergrund auf Organisations- und Personalentwicklung, so zeichnet sich ein - für Praktiker oft schwer verdauliches - Paradoxon ab: Einerseits geht es darum, Strukturen zu stabilisieren, was unverzichtbar ist, wenn eine größere Zahl von Mitarbeitenden

\section{"Leitungskräfte müssen ein Paradoxon bewältigen: Strukturen einerseits stabilisieren und andererseits flexibel halten"}

Die Art des Innenausbaus der Einrichtung und des Trägers ist maßgebend dafür, ob es zu schaffen ist, dem Unternehmen im Wettbewerbsfeld - also im Vergleich mit anderen Anbietern - eine zumindest mittelfristig (für drei bis fünf Jahre) gefestigte Position zu sichern. Neben technischen Faktoren (z. B. Geräte und andere Arbeitsmittel, Räume, Bauten) basiert der Innenausbau auf zwei Eckpfeilern:

- Die Organisationsstruktur besteht aus dem System der Standards, Normen und Wertorientierungen, mit deren Hilfe die Ordnung der Kommunikationswege und Kommunikationsinhalte sowie der Leitungsaufgaben und Leitungsbefugnisse gestaltet und stabilisiert werden. Ebenso geht es um die Strukturierung der Arbeitsprogramme, also der nach außen gerichteten Angebote und der sie unterstützenden internen Services wie Finanz- und Rechnungswesen, Facility Management etc. (sog. Supportservices).

- Die Personalstruktur beruht auf den Standards, Normen und Wertorientierungen, die ein geordnetes und über längere Zeit hinweg kooperieren sollen, um adressatengerechte Angebote zu erbringen. Andererseits kommt es darauf an, wie der Begriff Entwicklung sagt, diese Strukturen sowie die in ihrem Rahmen handelnden Personen immer wieder flexibel auf neue Umfeldanforderungen (Kundenwünsche und Adressatenanliegen, Rechtsvorschriften usw.) einzustimmen und auszurichten. Genau wegen dieses scheinbaren Paradoxons gehört das Koordinieren von Organisationsentwicklung und Personalentwicklung zu den spannendsten Aufgaben bei der Gestaltung des Innenausbaus eines Unternehmens.

\section{Warum Lenkung für Organisations- und Personalentwicklung nötig ist}

Wenden wir uns genauer den beiden zentralen Komponenten des Innenausbaus zu, die einerseits $\mathrm{zu}$ stabilisieren und andererseits immer wieder flexibel anzupassen sind. Beides gelingt im Unternehmen ohne aufwendige Reibungsverluste nur dann, wenn möglichst viele gut abgestimmt agieren. Dieses Abgestimmt-Sein lässt sich am besten durch eine Kombination von Wort und Bild erreichen, weil die Gehirnforschung zeigt, dass unser Gehirn ein »märchenerzählendes « Organ ist (Ramachandran 2011). Bewährt hat sich bei unserer Arbeit in der Praxis das Bild eines Fahrrads mit drei Rädern (vgl. Abbildung).

Das Antriebsrad Motivation und daraus resultierende Handlungen funktioniert nicht von selbst, sondern erfordert Motivierung. Aktivitäten des Motivierens können im Gehirn der Mitarbeitenden angesiedelt sein, also selbstmotivierend wirken, oder von außen auf das Gehirn einwirken (extrinsische Motivierung). In beiden Fällen geht es darum, eine Balance zu schaffen und immer wieder neu auszutarieren zwischen Anerkennung und Kritik, Überforderung und Unterforderung, Stabilität und Wandel sowie zu viel und $\mathrm{zu}$ wenig Sicherheit.

Sind die Aktivitäten des Motivierens gut dosiert und koordiniert, resultiert daraus ein hohe Arbeitsmotivation, anders gesagt: starke innere Schubkräfte für das Arbeiten und die Zusammenarbeit mit anderen. Diese Schubkräfte sind maßgebend dafür, ob die im Unternehmen anfallenden Aufgaben schleppend erledigt oder zügig erfüllt werden - ob also das Unternehmens-Fahrrad im Stau steht oder rollt.

Maßnahmen der Organisationsentwicklung und der Personalentwicklung gehören zu den Faktoren, die motivierend oder demotivierend wirken. Diese Maßnahmen bedürfen - wie alle anderen Aktionsprogramme des Unternehmens - einer Lenkung. Nicht vergessen: Wer Fahrrad fährt, ohne ein Ziel zu haben und ohne seinen Kurs gemäß Beschaffenheit der Wege immer wieder zu justieren, dreht sich schlimmstenfalls im Kreis und fällt auf die Nase. Im Unternehmen sollte das durch die Lenkung verhindert werden. Sie besteht aus der Kombination von Leiten, Steuern und Führen (Hegner 2010). In Stichworten:

- Zum Leiten gehört es, Ziele, also angestrebte Ergebnisse, aus Anforderungen des Umfelds abzuleiten sowie klar $\mathrm{zu}$ formulieren und $\mathrm{zu}$ begründen. Ergänzend sind daraus Leitlinien abzuleiten, also Konkretisierungen hinsichtlich der Ziele und Teilziele sowie der wichtigsten Aktionsprogramme, um sich den Zielen anzunähern. 


\section{Integrierte Organisations- und Personalentwicklung: vorwärts mit drei Rädern}

Das Aktionsfeld ist fast immer holprig! Da hilft stromlinienförmige Eleganz wenig!

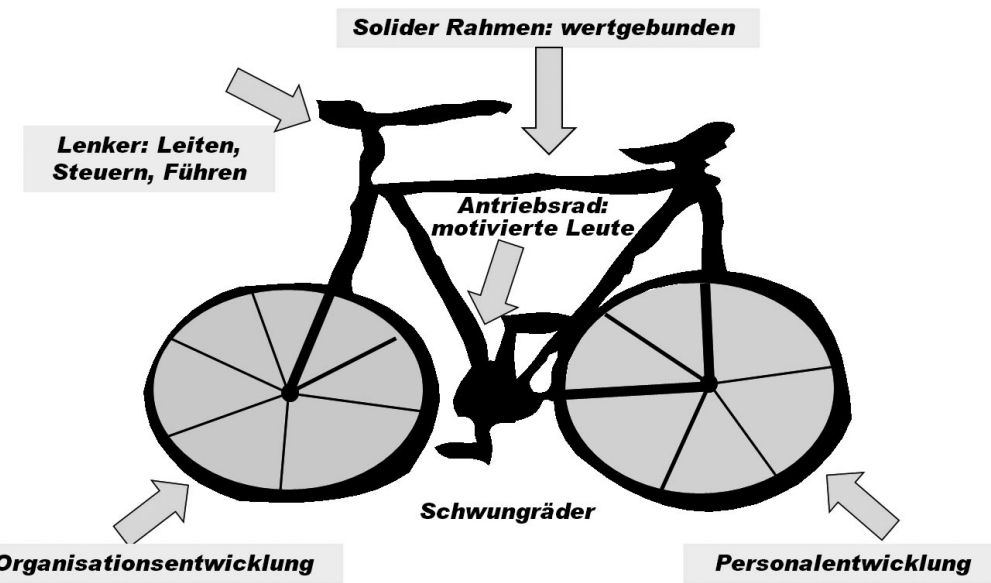

Organisationsentwicklung und Personalentwicklung können sinnbildlich als die beiden Schwungräder gesehen werden, die den Innenausbau des Unternehmens immer wieder in geordnete Bewegung versetzen. Die Motivation der Mitarbeitenden ist dabei das Antriebsrad.

- Steuern besteht darin, immer wieder einen Abgleich zwischen angestrebten und - jeweils aktuell - tatsächlich erreichten Ergebnissen durchzuführen. Darauf folgen Maßnahmen, um die ständige Annäherung von Sollund Ist-Zustand zu bewirken. Dazu gehört die Beantwortung folgender Fragen: Sind die Ziele noch realitätsgerecht oder müssen sie modifiziert werden? Sind die formulierten Leitlinien - inklusive fachliche Standards und soziale Normen - noch zweckmäßig und angesichts veränderter Rahmenbedingungen weiterhin praktikabel?

- Maßnahmen des Leitens und Steuerns laufen ausschließlich dann nicht ins Leere, wenn es gelingt, die als richtig angesehenen Dinge verständlich $\mathrm{zu}$ machen und motivierend $\mathrm{zu}$ vertreten. Damit sind wir beim Führen. Hier geht es darum, Richtwerte (Ziele, angestrebte Ergebnisse) und Leitlinien für Prozesse sowohl in die Sprache der einzelnen Mitarbeitergruppen zu übersetzen als auch für die unterschiedlichen Mitarbeitergruppen attraktiv zu machen. Dazu gehört: Entscheidungen begründen, Anregungen geben, fachliche Beratung durchführen, aufmerksam zuhören, Mitarbeitende in Veränderungsprozesse einbeziehen und $\mathrm{zu}$ Mitgestaltenden machen, usw.
Leiten, Steuern und Führen geben der Organisations- und Personalentwicklung sowohl die notwendige Orientierung als auch die angesichts veränderter Umfeldbedingungen unverzichtbare Justierung. Das hält die beiden Schwungräder des Unternehmens-Fahrrads auf klarem Kurs. Diesbezügliche Maßnahmen wiederum sind bei der Alltagsarbeit durch gut durchdachte Checklisten und Aktionspläne zu hinterlegen, denn ohne Landkarte oder gar Geländekarte begibt sich kein umsichtiger Radwanderer auf eine Reise in holprigem Fremdgelände.

\section{Was Organisations- und Personalentwicklung leisten muss}

Bildlich gesagt fungiert die Organisationsentwicklung als vorderes Schwungrad. Es wird gehalten von der Vorderradgabel mit zwei Rohren. Damit sind die beiden wichtigsten Arbeitsprogramme der Organisationsentwicklung angesprochen:

- Haupt- und Teilprozesse in den einzelnen Arbeits- und Geschäftsbereichen durchleuchten und verbessern. Das gelingt nur durch eine klar gelenkte Blick-und Aktionsrichtung: Qualität bei Sachgütern und Dienstleistungen steigern, Termintreue gewährleisten, Arbeitsproduktivität erhöhen, Nutzungszeit und Nutzungsgrad bei
Plätzen, Betten, Aggregaten steigern, Energie- und Materialverbrauch senken, aber auch negativen Dauerstress abbauen und dadurch Humanressourcen schonen sowie motivierende Arbeitsbedingungen schaffen und Fachkräftelücken vermeiden.

- Ergänzt wird die Makro-Ebene der Prozessgestaltung und Prozessentwicklung durch die Mikro-Ebene der Überprüfung und Gestaltung des Stellenzuschnitts. Hier geht es darum, hinsichtlich der Arbeitsstellen, die in die Prozesse eingebettet sind und an denen die Prozesse alltäglich realisiert werden, Folgendes ziel- und ergebnisgerichtet auszuwählen und je Stelle zu ordnen: zu bearbeitende Merkmale von Kunden, Patienten, Klienten, Nutzern etc. (z. B. bei Anamnese, Diagnose, Therapie, Beratung, Ausbildung) oder von Sachen (z. B. bei der Raum- oder Materialbeschaffung); zweckmäßige Arbeitsmittel und Arbeitsinstrumente; geeignete Arbeitsmethoden; Abfolge von Arbeitsschritten im Tagesgeschäft; zu erreichende Arbeitsergebnisse (inklusive der Wirkungen auf die Nutznießer, an die sie gerichtet sind).

Parallel zur Beschreibung des Stellenzuschnitts erfolgt die Beschreibung der Anforderungen hinsichtlich der benötigten Personalmerkmale. Mit dem Stichwort »Personalmerkmale « nähern wir uns dem Antriebsrad (Motivierung und Motivation), das seinerseits eng mit dem hinteren Schwungrad verbunden ist, also mit der Personalentwicklung. Somit gehört die Gestaltung und ständige flexible Weiterentwicklung der Stellenzuschnitte und Anforderungsprofile zu den Aufgaben, die in enger Abstimmung zwischen Organisationsentwicklung und Personalentwicklung erledigt werden müssen. Das gilt besonders für folgende Aufgaben, die direkt mit beiden Aufgabenfeldern verbunden sind:

- Karriere- und Laufbahnplanung, deren Transparenz für die Motivierung der Mitarbeitenden ebenso wichtig ist wie für die Anwerbung neuen Personals

- Nachfolgeplanung, was angesichts der demografischen Überalterung immer wichtiger wird

- Qualifikationsbedarf ermitteln und fortschreiben, was sowohl für das 
Einarbeiten neuer Mitarbeitender als auch für die Weiterqualifizierung bisheriger Mitarbeiterinnen und Mitarbeiter ausschlaggebend ist, damit die wechselnden Stellenanforderungen passgerecht ausgefüllt werden können

- Koordinieren der Gestaltung von Betriebszeiten (z. B. Öffnungs- und Ansprechzeiten) und Arbeitszeiten, wobei die Vereinbarkeit von Berufsund Privatleben in unterschiedlichen biografischen Phasen eine zunehmende Rolle spielt, beispielsweise bezüglich sowohl der Betreuung von Nachwuchs als auch der Pflege hilfebedürftiger Angehöriger oder Freunde.

Das alles sind - ebenso wie die Personalbeschaffung - Standardaufgaben der Personalarbeit - abgestimmt mit der Organisationsentwicklung. Innerhalb der Personalarbeit werden durch die Personalentwicklung zwei Akzente gesetzt bildlich als Hinterradgabel:

- Humanressourcen schonen, das heißt: Verschwendung und unnötigen Verschleiß von Arbeitszeit sowie psychischen und physischen Energien vermeiden, beispielsweise durch reduzierte Administrationsaufgaben bei Fachkräften; Gesundheit schützen, beispielsweise durch ergonomisch optimierte Arbeitsmittel; negativen Dauerstress aufgrund fehlender Balance von qualifikatorischer und zeitlicher Unter- und Überforderung auf ein Mindestmaß beschränken; Lebens- und Berufserfahrung bewahren, indem ältere Mitarbeitende nicht, wie "altes Eisen « behandelt werden.

- Humanpotenziale entfalten, das heißt: zum einen bisher gar nicht oder nur teilweise genutzte Kenntnisse, sensorisch-motorische Fertigkeiten und psychosoziale Fähigkeiten der vorhandenen Mitarbeiter zur vollen Entfaltung zu bringen, beispielsweise durch Gestaltung der inner- und außerbetrieblichen Fort- und Weiterbildung; der Rekrutierung von neuem Personal auch auf solche Personengruppen achten, die - wie beispielsweise Immigranten, Schul- oder Ausbildungsabbrecher - bis dahin eher vernachlässigt wurden, weil es genügend Alternativen gab, solange die geburtenstarken Jahrgänge im erwerbsfähigen Alter waren. Beides ist unverzichtbar, um bei der Angebotsentwicklung auf dem fachlich neuesten Stand zu sein, bei der Arbeitsproduktivität besser zu werden, ohne die Mitarbeitenden zu überfordern, sowie die Angebotsqualität - gerade bei scharfem Wettbewerb-immer wieder ein kleines Stück zu verbessern.

Die Schonung der »Humanressourcen« und die Entfaltung der »Humanpotenziale « müssen Hand in Hand erfolgen, weil beide Aufgabenfelder auf die gleichen Personalmerkmale gerichtet sind, wenn auch mit unterschiedlichem Blickwinkel: Kenntnisse (kognitive Merkmale), sensorische und motorische Fertigkeiten, psychische und soziale Fähigkeiten, aber auch Anteile der Lebenszeit.

Der letztgenannte Aspekt wird in dem Maße immer wichtiger, wie es angesichts der demografischen Überalterung und »Unterjüngung « zunehmend darauf ankommt, Männer und Frauen sowohl mit Elternaufgaben als auch mit Pflegeaufgaben für die eigenen Eltern und Angehörigen nicht einfach auf Halbzeitjobs oder geringerwertige Aufgaben abzuschieben. Damit würden Humanpotenziale ungenutzt bleiben. In die gleiche Falle - aus umgekehrter Richtung - laufen diejenigen Unternehmen, bei denen der Prozess- und Stellenzuschnitt so gestaltet ist, dass Männer und Frauen mit Familienaufgaben überfordert werden und sich wegen negativem Dauerstresses krankmelden müssen (Gerbracht/Hegner/Kramer 2011).

\section{Was tun?}

Ziehen wir Bilanz: Das Bild des Unternehmens-Fahrrads macht deutlich, wie wichtig es ist, zum einen die Organisationsentwicklung und die Personalentwicklung zu koordinieren sowie zum Zweiten beide eng mit den Lenkungsaufgaben (Leiten, Steuern, Führen) und mit der strategischen Positionierung im Wettbewerbsfeld zu verbinden. Diese anspruchsvollen Aufgaben gelingen leichter, wenn man Konsequenzen daraus zieht, dass bei einem Fahrrad die drei Räder und die Lenkung durch einen - hoffentlich stabilen - Rahmen verbunden sind. Was bedeutet das für Unternehmen?

Es richtet die Aufmerksamkeit darauf, dass Organisationsentwicklung und Personalentwicklung nur dann klare und für alle erkennbare Konturen erhalten, wenn sie in einen Rahmen gemeinsamer Wertorientierungen eingebunden sind. Wertorientierungen und die dadurch geschärften Konturen wirken - ohne viele Worte - handlungsleitend, was das koordinierte Handeln einer Vielzahl von Personen erleichtert (Hegner 2009, 2010).

Akzente solcher Wertorientierungen lassen sich aus humanistischen Überzeugungen ebenso gewinnen wie aus religiösen oder ökologischen Leitideen. Die praktische Wirksamkeit wird erhöht, wenn sie nicht nur in ein Unternehmensleitbild eingebaut sind, sondern aus ihnen tatsächlich konkrete Folgerungen für Aktionsprogramme gezogen werden, also vor allem für das Zusammenspiel von Maßnahmen der Organisationsentwicklung und der Personalentwicklung.

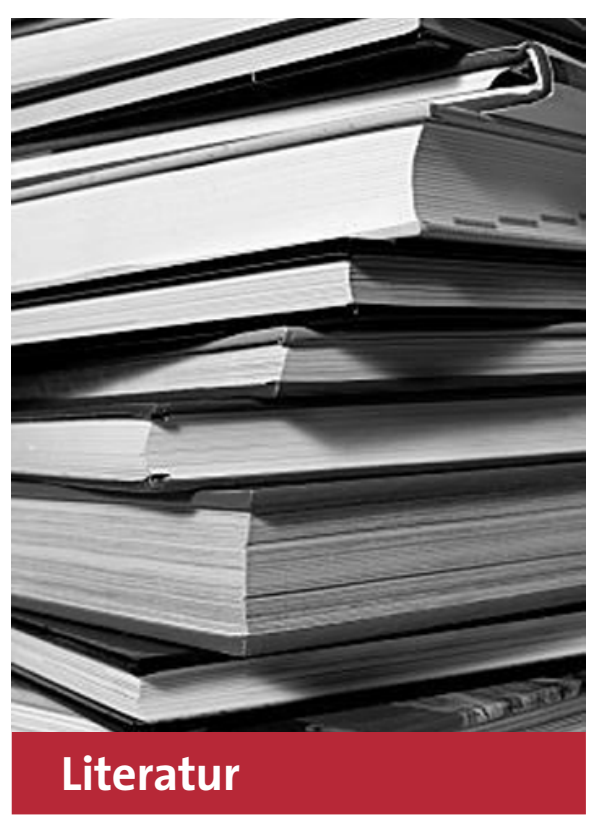

Gerbracht, Petra/Hegner, Friedhart/Kramer, Ulrich: Ausfallzeiten, Hauruck-Aktionen sind keine Lösungen. In: SOZIALwirtschaft. Zeitschrift für Sozialmanagement Jg. 21 (2011), Heft 6, S. 12-15.

Hegner, Friedhart: Leiten, steuern, führen. In: SOZIALwirtschaft. Zeitschrift für Sozialmanagement Jg. 20 (2010), Heft 4, S. 32-34.

Hegner, Friedhart: Mehr Freiraum durch Disziplin: Was leistet ein Unternehmensbild in der Wohlfahrtspflege? In: H.-J. Dahme/N. Wohlfahrt (Hg.), Systemanalyse als politische Reformstrategie. Wiesbaden: Verlag VS (2010), S. 335-346.

Ramachandran, Vilayanur Subramanian: The Tell-Tale Brain. London: Heinemann 2011. 